\title{
Attenuated phase-shifting photomasks fabricated from Cr-based embedded shifter blanks
}

\author{
F. D. Kalk, ${ }^{*}$ R. H. French, ${ }^{* *}$ H. U. Alpay, ${ }^{* *}$ G. Hughes, ${ }^{* * *}$ \\ *** DuPont Photomasks Inc., and DuPont Co. *Electronic Materials and ${ }^{* *}$ Central Research \\ Wilmington, DE 19880-0356 USA
}

\begin{abstract}
I-line $(365 \mathrm{~nm})$ and G-line $(436 \mathrm{~nm})$ attenuated phase shifting photomasks have been developed using single layer $\mathrm{Cr}$ based photoblanks. The absorber layer has a composition gradient that allows the desired transmission to be tuned while maintaining control over reflectivity and phase shift. These photoblanks are manufactured in existing facilities, and masks are processed much like conventional opaque $\mathrm{Cr}$-based materials. They can be inspected and repaired on current equipment with slight modifications. Printing has been demonstrated on current generation steppers. Deep UV extendability of these materials is also being studied, with a 5\% Deep UV (248 nm) single layer photoblank chemistry already demonstrated.
\end{abstract}

\section{INTRODUCTION}

Mask fabrication complexity is a commercialization barrier for phase shifting photomasks. The mask maker must perform new process steps (e.g., $\mathrm{SiO}_{2}$ etching), certify the phase shift of the finished product, and guarantee zero defects. Mask design complexity may also adversely impact data preparation and mask inspection. While the weak shifter approaches (rim, attenuated) do not afford as much resolution and/or process latitude as the strong approaches (alternating, subresolution, chromeless), they are applicable to all pattern geometries. Attenuated phase shifting photomasks offer the added potential for both simple design and fabrication. ${ }^{1}$

The ideal attenuated phase shifting photomask would have a single absorber layer combining the desired transmission, reflectivity, and $180^{\circ}$ phase shift at the lithographic wavelength. ${ }^{2}$ The mask would be processed with existing mask shop tools and chemistries: writing would be accomplished with either electron beam or scanning laser; absorber etching would be either wet or dry; inspection and repair would be performed on existing tools with perhaps slight modifications to accommodate the unique mask properties.

Our approach to atttenuated phase shifting photomasks is to produce a Cr-based embedded shifter photoblank (i.e., the $180^{\circ}$ phase shift is built into the absorber) with a graded chemistry. Graded Cr-based photoblanks satisfying a wide range of requirements can be produced and processed in a manner similar to conventional Cr-on-quartz blanks.

\section{PHOTOBLANK FABRICATION}

The Cr-based embedded shifters described here are produced by batch inline reactive sputtering. In this technique (shown in figure 1), a large number of trays, each holding multiple fused silica substrates, are loaded into a load magazine and pumped into high vacuum. Trays are then driven sequentially through a process chamber where the film is deposited by a bank of sputtering cathodes. The coated trays accumulate in an unload magazine. After coating, the process chamber is isolated from the magazines, the magazines are vented to atmosphere, and the trays are unloaded. Over one hundred $6 "$ plates can be coated in a single batch, although batch sizes usually range between 25 and 50 plates. The material properties are tailored by varying the cathode powers and gas mixture.

Cr-based films' flexible properties are suitable for several applications. Materials have been developed for both I-line and G-line applications. Initially, I-line embedded shifter materials were developed for the 5-15\% transmission range. As wafer fabs have gained experience with attenuated phase shifting photomasks, emphasis has increased on the 6-10\% range. 


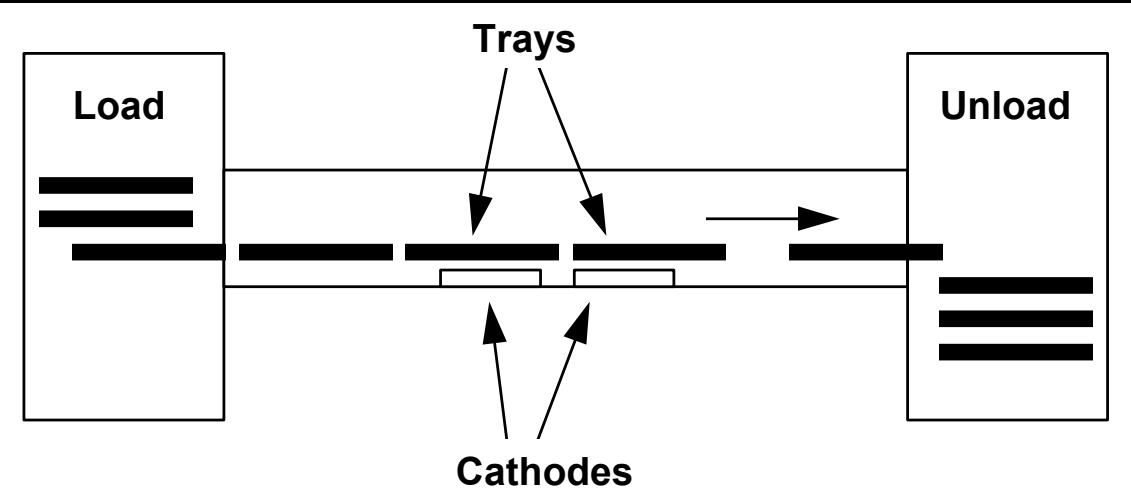

Fig. 1. Schematic diagram of the sputttering tool layout.

\section{METROLOGY TOOLS}

Graded Cr-based films require advanced optical metrology. For example, phase shift cannot be calculated from a single $[\mathrm{n}, \mathrm{k}]$ pair at the lithographic wavelength. For this reason, phase sensitive interferometry, variable angle spectroscopic ellipsometry (VASE), optical spectroscopy, stylus profilometry, Rutherford Backscattering Spectrometry (RBS), and Sputtered Neutrals Mass Spectrometry (SNMS) are employed to synthesize detailed internal film models and verify their accuracy. These techniques support rapid design and development of attenuated phase shifting photoblanks at I-line, G-line, and DUV wavelengths.

\subsection{Direct Phase Shift Measurement}

Phase sensitive interferometry is used to measure phase shift directly at three or four wavelengths. Figure 2 depicts the dual beam interferometer's optics. The instrument incorporates a mirror driven by a piezoelectric transducer to introduce a linearly varying phase shift in one beam. Spot separation at the mask is adjusted by the relative tilt between the beams, allowing the use of various numerical apertures. The mask is translated so the two beams cross an edge patterned in the film. The beams are recombined to form an interference pattern at the photodiode detector. As each spot crosses the film edge, the phase of the signal changes by an amount equal to the film's phase shift at the incident wavelength.

Figure 3 shows $488 \mathrm{~nm}$ interferometer scan data for a typical I-line embedded shifter film. Usually four scans across the phase edge (encompassing 240 data points) are averaged to enhance measurement precision. A second order curve is fit to the multiple wavelength data to produce a spectral phase shift plot.

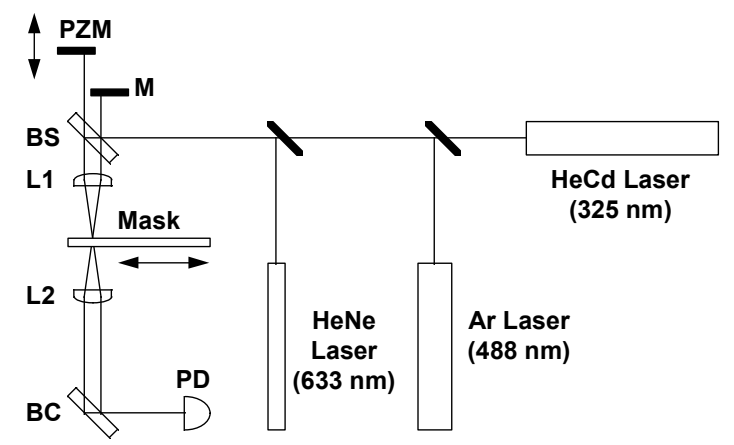

Fig. 2. Phase sensitive interferometer optical layout . M: mirror; PZM: piezoelectrically driven mirror; BS: beam splitter; L1, L2: lenses; BC: beam combiner; PD: photodiode.

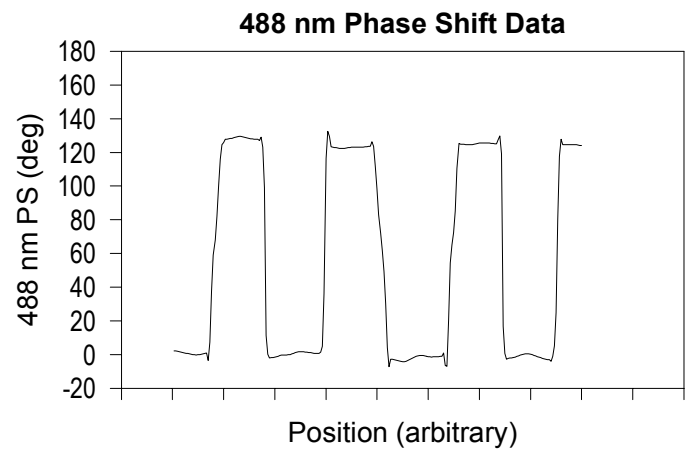

Fig. 3. $488 \mathrm{~nm}$ interferometer scan data for a typical single layer absorber with $\sim 180^{\circ}$ I-line phase shift. 


\subsection{Detailed Film Modelling}

The interferometer provides only direct phase shift information. A seed model of the film's depth-dependent optical properties is developed using stylus profilometry, RBS, and SNMS data (RBS is of limited utility because it has poor sensitivity for the lighter elements). Figure 4 shows SNMS data for an I-line embedded shifter (stylus profilometry indicated the film thickness to be $122.5 \mathrm{~nm}$ ). The seed model consists of an [n,k] depth profile based on the oxygen profile (the nitrogen and carbon profiles mirror oxygen). The details of the model are adjusted to fit optical spectroscopy and VASE data. ${ }^{3}$ Figure 5 shows spectral transmission data for the film profiled in figure 4.

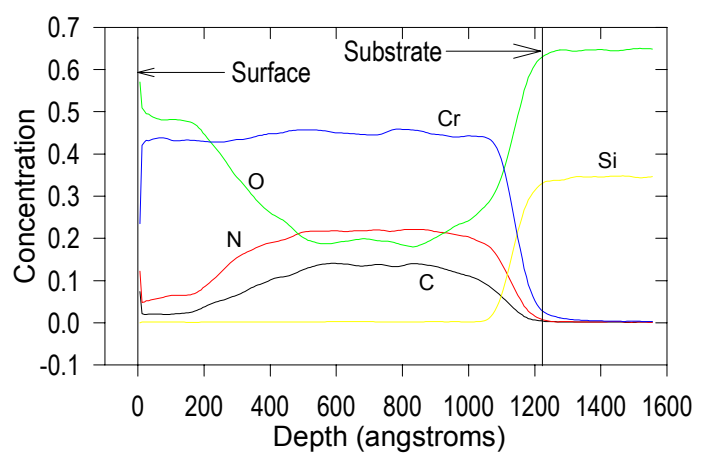

Fig. 4. SNMS elemental depth profile data for an I-line embedded shifter material.

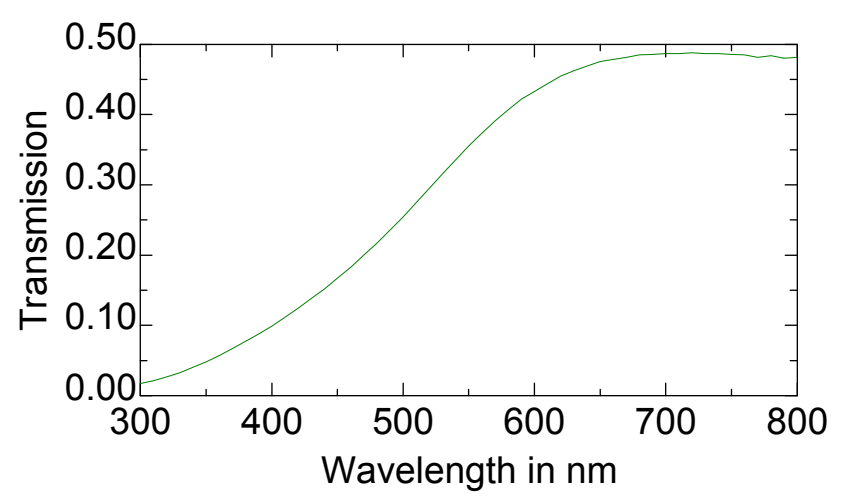

Fig. 5. Spectral transmission for the film in fig. 4.

Figure 6 shows the VASE configurations used to constrain the film model. Data are gathered at $75^{\circ}$ incidence angle. The ellipses indicate that the polarization changes upon transmission or reflection. Backside ellipsometry, in which the illumination is substrate-incident, helps to interrogate the substrate-film interface. When using a substrate with a smooth back surface, it is critical to quantify the relative contributions of the multiple detected beams, either reflected (fig. 6b) or transmitted (fig. 6c).

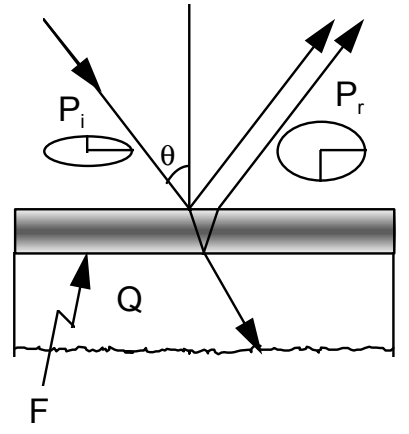

(a)

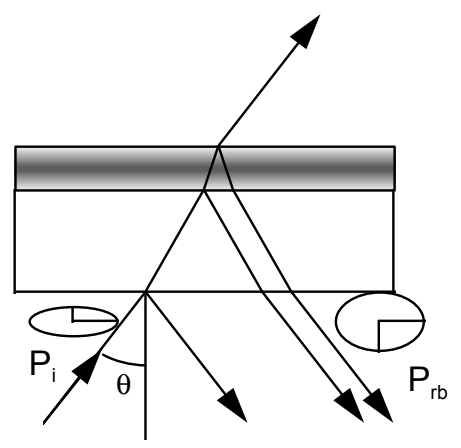

(b)

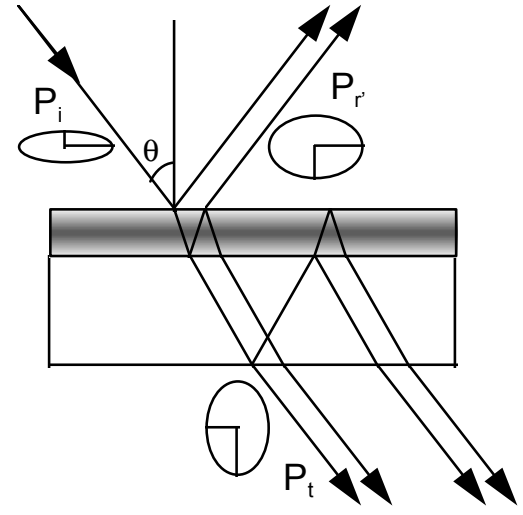

(c)

Fig. 6. Ellipsometric geometries used for analysis of graded Cr-based materials: (a) "conventional" reflection ellipsometry with film-incident illumination and a depolarizing substrate back surface; (b) reflection ellipsometry with substrate-incident illumination; (c) reflection and transmission ellipsometry with a smooth substrate back surface. S: substrate; F: film; $\theta$ : angle of incidence; $\mathrm{P}_{\mathrm{i}}$ : incident illumination; $\mathrm{P}_{\mathrm{r}}$ : reflected radiation in geometry $(\mathrm{a}) ; \mathrm{P}_{\mathrm{rb}}$ : reflected radiation in geometry (b); $\mathrm{P}_{\mathrm{t}}$ : transmitted radiation in geometry $(\mathrm{c}) ; \mathrm{P}_{\mathrm{r}}$ : reflected radiation in geometry $(\mathrm{c})$.

The optical properties of graded Cr-based embedded shifters can be described effectively with the Bruggeman formalism of the Effective Medium Approximation (EMA). ${ }^{4}$ In the Bruggeman EMA, the material at any depth is assumed to be a combination of two (or more) constituent materials which have wavelength-dependent optical properties. The model specifies these constituents and their relative proportions as a function of film depth. The film is not constrained to consist of purely 
one constituent material at any depth. Excellent fits can be achieved by using polynomial expressions to describe the constituents' $[\mathrm{n}, \mathrm{k}]$ wavelength dependence. The model then calculates the depth dependence of the optical properties.

Figure 7 is a plot of the I-line depth profiles for $\mathrm{n}$ and $\mathrm{k}$ derived from the model. The profiles are consistent with the SNMS data in figure 4; both $\mathrm{n}$ and $\mathrm{k}$ will be higher for lower oxygen concentration. Figure 8 shows the spectral transmitted phase shift calculated with the model and the second-order fit derived with the phase sensitive interferometer. The fits agree within $6^{\circ}$ over the wavelength range from 325 to $635 \mathrm{~nm}$ and confirm the model's validity.

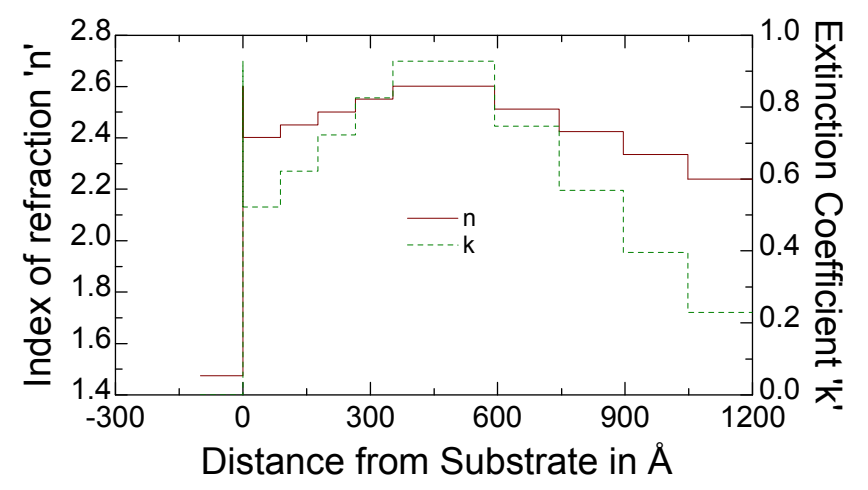

Fig. 7. Model-generated depth profiles for refractive index and extinction coefficient for the film profiled in figure 4.

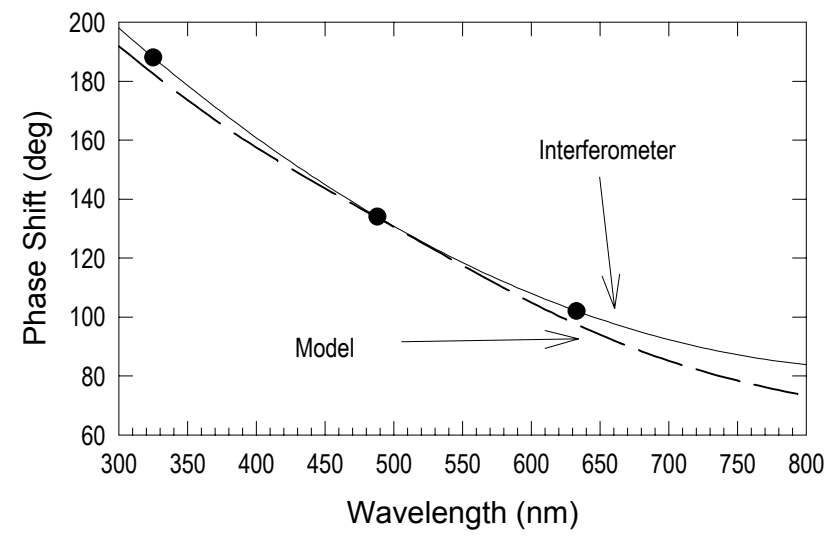

Fig. 8. Spectral transmitted phase shifts determined by the optical model and the phase sensitive interferometer.

\section{Cr-BASED EMBEDDED SHIFTER PHOTOBLANKS}

\subsection{I-line Material}

Cr-based I-line materials have thicknesses in the $110-130 \mathrm{~nm}$ range. The example in section 3 is a candidate I-line embedded shifter material. Its I-line transmission is about $6.5 \%$ relative to a fused silica reference and is toward the lower end of interest. In addition to the properties shown in section 3 , it exhibits the following properties:

Table 1. Additional properties of the I-line material shown in section 3.

\begin{tabular}{|c|c|}
\hline Reflectivity $(365 \mathrm{~nm})$ & $14 \%$ \\
\hline Wet Etch Time-to-Clear & 65 seconds \\
\hline Thickness & $120 \mathrm{~nm}$ \\
\hline Transmission $(488 \mathrm{~nm})$ & $22 \%$ \\
\hline Transmission $(633 \mathrm{~nm})$ & $45 \%$ \\
\hline
\end{tabular}

It is possible to optimize individual material properties, as demonstrated in Table 2, in which the properties of two materials (designated A and B) are compared. By adjusting the composition and gradient of the film, the wet etch time-toclear of material B can be increased over that of material A, while the two materials' optical properties are nearly identical. In this example, wet etch time was measured in a commercially available etchant based on nitric acid. ${ }^{5}$ Relevant data are also shown for conventional opaque Cr-based photoblanks.

Table 2. Properties of two Cr-based I-line embedded shifters, with Cr-on-Qz shown for reference.

\begin{tabular}{|c|c|c|c|c|c|c|c|}
\hline \multirow[t]{2}{*}{ Material } & \multirow{2}{*}{$\begin{array}{c}\text { Thickness } \\
\text { (nm) }\end{array}$} & \multirow{2}{*}{$\begin{array}{c}\text { PS } \\
(365 \mathrm{~nm})\end{array}$} & \multicolumn{3}{|c|}{ Transmission (quartz reference) } & \multirow{2}{*}{$\begin{array}{c}\text { Reflectivity } \\
(365 \mathrm{~nm})\end{array}$} & \multirow{2}{*}{$\begin{array}{l}\text { Wet Etch } \\
\text { Time (s) }\end{array}$} \\
\hline & & & $365 \mathrm{~nm}$ & $488 \mathrm{~nm}$ & $633 \mathrm{~nm}$ & & \\
\hline Cr-on-Qz & 125 & --- & $0.1 \%$ & --- & -- & $14 \%$ & 70 \\
\hline Mat'l. A & 112 & $180^{\circ}$ & $6.3 \%$ & $23 \%$ & $49 \%$ & $15 \%$ & 30 \\
\hline Mat'l. B & 128 & $180^{\circ}$ & $6.4 \%$ & $24 \%$ & $49 \%$ & $14 \%$ & 65 \\
\hline
\end{tabular}




\subsection{G-Line Material}

Properties of a candidate G-line chemistry are shown in figure 9. At $436 \mathrm{~nm}$ wavelength, the material exhibits $189^{\circ}$ phase shift (i.e., the film is too thick) and 6.8\% transmission. Decreasing the thickness from $146 \mathrm{~nm}$ to $139 \mathrm{~nm}$ decreases the phase shift to $180^{\circ}$ and increases the transmission to $7.7 \%$. G-line embedded shifters covering the $2-20 \%$ transmission range can be produced by adjusting the process chemistry.

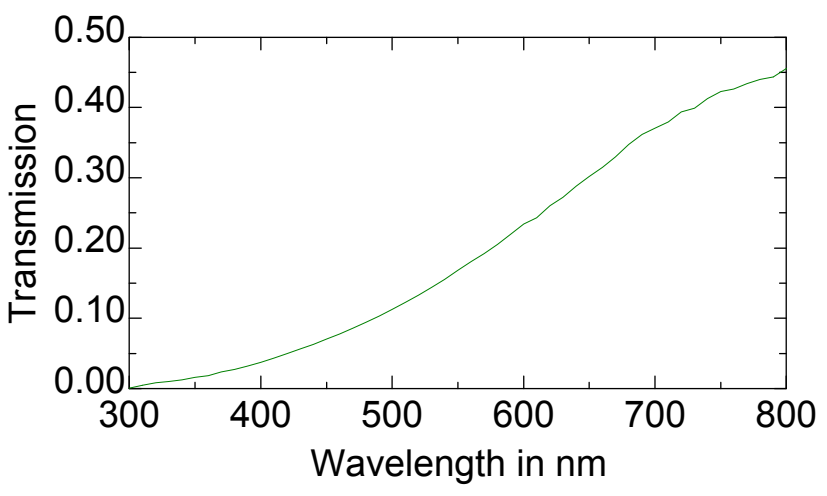

(a)

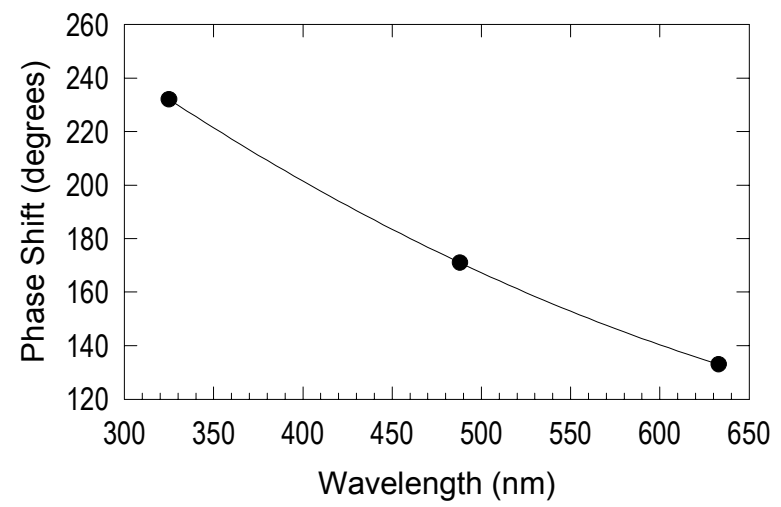

(b)

Fig. 9. Spectral transmission (a) and phase shift (b) of a candidate G-line embedded shifter material.

\subsection{DUV Material}

Figure 10 shows the spectral properties of a DUV candidate. The film thickness is $101.5 \mathrm{~nm}$, the $248 \mathrm{~nm}$ transmission is $3.6 \%$, and the $248 \mathrm{~nm}$ phase shift is $198^{\circ}$. Reducing the thickness to $92 \mathrm{~nm}$ decreases the phase shift to $180^{\circ}$ and increases the transmission to $4.9 \%$. This indicates that $\mathrm{Cr}$-based embedded shifters may be extendable to the DUV.

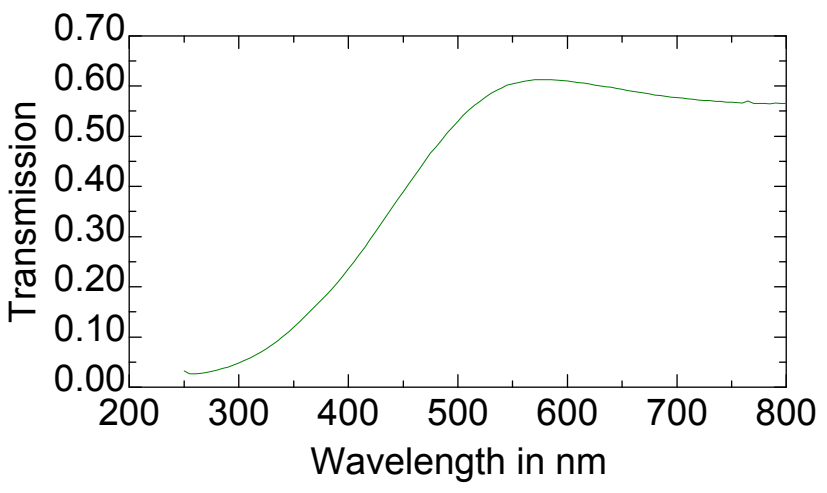

(a)

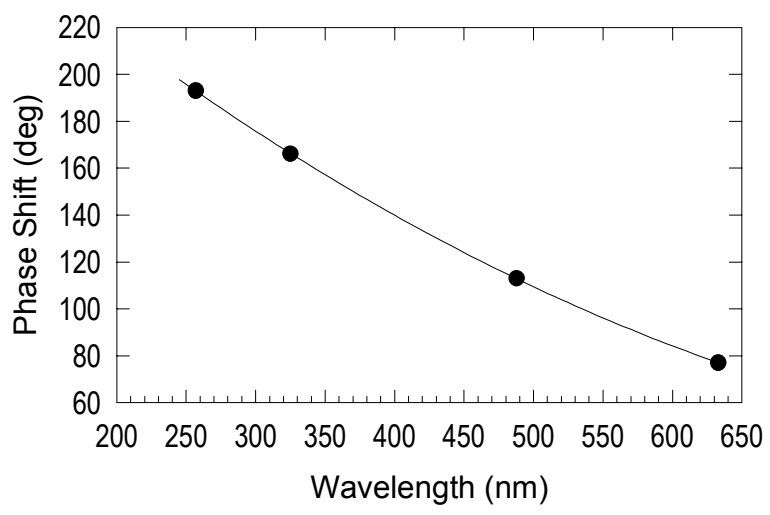

(b)

Fig. 10. Spectral transmission (a) and phase shift (b) of a candidate DUV embedded shifter material.

\subsection{Photoblank Manufacturability}

To ensure manufacturability, each photoblank's optical and defect properties must be certified. It is desirable to use the existing tool base. Transmission, reflectivity, and particle inspection can be measured as in conventional opaque photoblank manufacturing. Phase shift and pinhole inspection are somewhat more complicated and are reviewed below.

Phase sensitive interferometry provides fast and accurate phase shift measurement but requires a patterned film. Although nondestructive, ellipsometric phase shift determination is slow and increases material handling. A third approach is 
viable. Thickness error is the dominant film property variation within a batch (i.e. the film chemistry is constant). For the thickness nonuniformities typical of photoblank manufacturing, thickness, phase shift, and optical density are linearly related. Thus, phase shift for any plate can be determined by comparing its transmission to that of witness plates whose transmission and phase shift are measured. Figure 11 illustrates this: $488 \mathrm{~nm}$ phase shift is plotted as a function of $365 \mathrm{~nm}$ optical density for thirteen plates selected from an extended batch. The error bars represent $\pm 1 \sigma$ measurement uncertainty. Large O.D. and phase shift variations were purposely selected to illustrate the technique. For the straight line fit in the figure, $\mathrm{r}^{2}=0.9$, even though phase shift and optical density were measured at different wavelengths. Measuring at the same wavelength improves the fit. This technique has been combined with a post-patterning etch of the substrate to correct the phase shift to $180^{\circ}$ on attenuators that are nominally less than $180^{\circ}$. Initial production tolerances for $7 \%$ I-line material ( $5 "$ and $6 "$ plates) are $\Delta \mathrm{T}=$ $\pm 0.5 \%$ (i.e. $6.5 \% \leq \mathrm{T} \leq 7.5 \%), \Delta \mathrm{R}= \pm 0.5 \%$, and $\Delta \mathrm{PS}= \pm 3^{\circ}$ within a plate. Similar tolerances apply for nominal plate-toplate values.

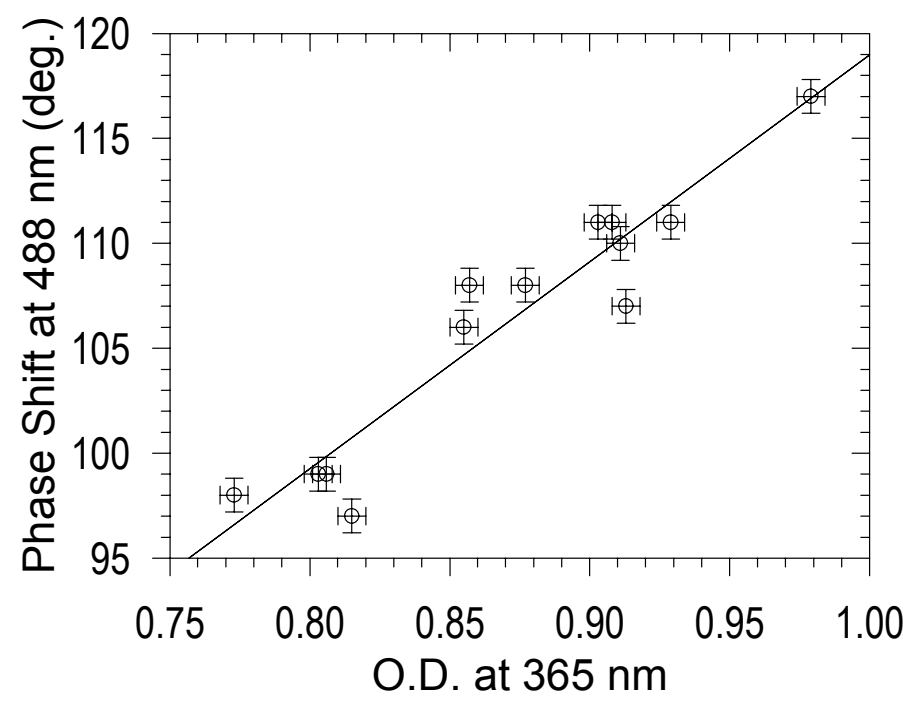

Fig. 11. $488 \mathrm{~nm}$ phase shift versus $365 \mathrm{~nm}$ optical density for several plates fabricated in an extended batch deposition.

The attenuated photoblank's partial transmission makes pinhole inspection difficult. Viable pinhole detection approaches include using a blank inspection algorithm on a commercial mask inspection tool ${ }^{6}$ and measurement of scattered transmitted light on a blank inspection tool. ${ }^{7}$ Pinholes smaller than $0.5 \mu \mathrm{m}$ are detectable.

\section{MASK FABRICATION}

\subsection{Mask Processing}

Cr-based embedded shifters process much like conventional Cr-based materials. The graded film structure provides a more conductive film than a non-graded layer, so electron beam writing is possible in many cases. The DUV material is not yet conductive enough to prevent electron beam charging. Laser writing is also used routinely with these materials.

Critical dimension control and edge roughness achieved with both patterning methods are comparable to those achieved with conventional opaque photoblanks. Wet and dry etching can be used, and etch times are comparable to those of conventional Cr-based photoblanks. Potential issues in dry etching include rate nonuniformity, CD nonuniformity, edge roughness, selectivity to both resist and the fused silica substrate, and pattern loading. Overetching can be employed to mitigate rate nonuniformity and pattern loading effects; typical overetch is about $20 \%$. Substrate etch selectivity is critical to avoid adding phase shift from substrate etching during attenuator overetch; selectivity is $>20: 1$ in the Cl-based etch gas employed for these embedded shifters. As much as $100 \%$ overetch on Cr-based embedded shifter materials does not degrade $\mathrm{CD}$ uniformity and edge roughness; $30 \%$ overetch introduces less than $2^{\circ}$ phase shift variation.

F. D. Kalk, R. H. French, H. U. Alpay, G. Hughes, SPIE Proceedings Vol. 2254, Photomask Japan 94. 


\subsection{Inpsection and Repair}

Inpsection systems require light calibration to inspect attenuated masks. KLA 200 Series systems operate at $565 \mathrm{~nm}$ wavelength with about $25 \%$ upper transmission limit, adequate for inspection of the G-line material shown in figure 9. KLA 300 Series systems operate at $488 \mathrm{~nm}$ with an upper transmission limit greater than $50 \%$, adequate for inspection of G-line and I-line materials. Preliminary 300 Series work with DUV material (figure 10) is positive. KLA 300 sensitivity to programmed defects on $10 \%$ I-line masks is comparable to its sensitivity on conventional binary masks, as shown on a Verimask 1045 pattern in figure 12.

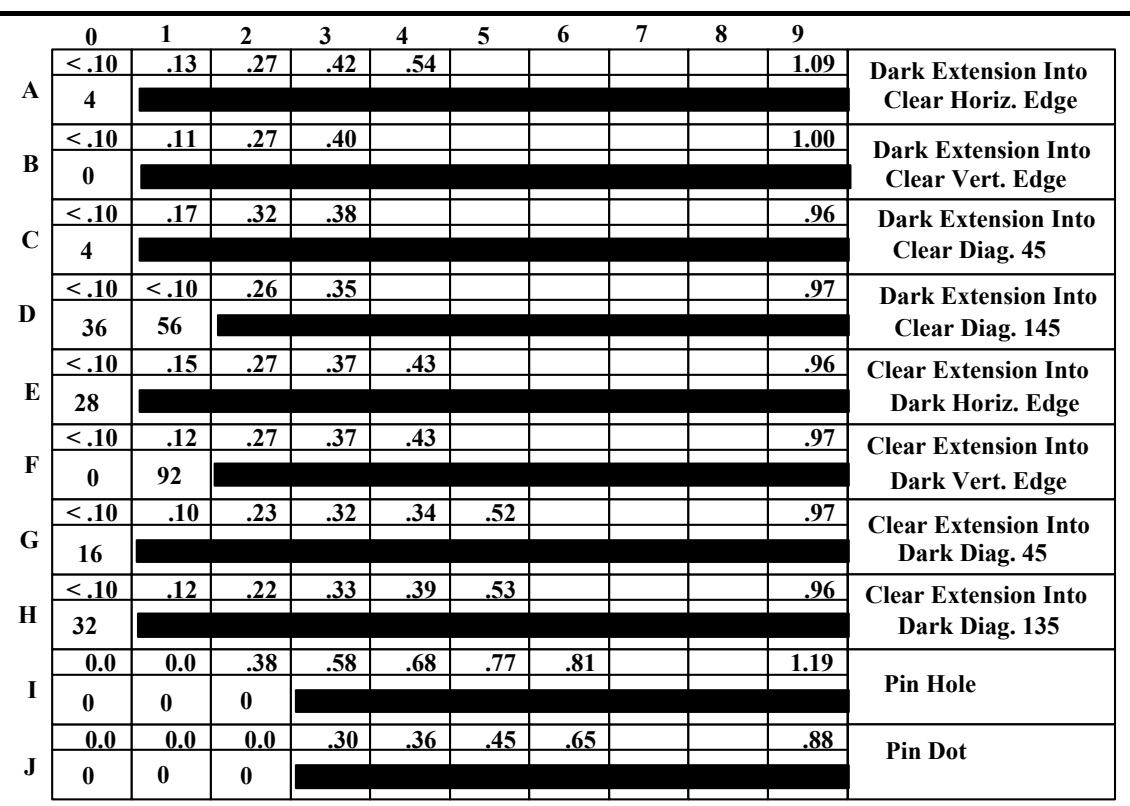

\section{Example}

\begin{tabular}{|r|l}
\hline .34 & Defect Size \\
\cline { 1 - 1 } 100 & KLA 331-PS Capture Rate, \% \\
\hline
\end{tabular}

For Defect Size Not Indicated:

(blank) Defect visible, not measured

Defects measured on Vickers Microscope

Attenuated-Embedded Verimask 1045 Inspection Reticle

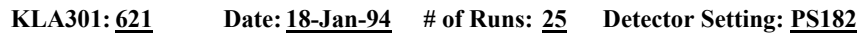

Mode: $\underline{\text { Die-Die }}$

Fig. 12. Inspection report for Verimask 1045 made with an I-line embedded shifter.

Pinspot removal in conventional $\mathrm{Cr}$ and embedded shifters is similar. Embedded shifter pinhole repair is more difficult because the repair must match the absorber's transmission and phase shift. Laser and focussed ion beam pinhole repair strategies are effective. Addition of gases appropriate for gas phase deposition of the repair material is required, and conventional repair tools are readily adapted.

Initial laser repair studies were performed on contact arrays containing programmed defects, as shown in figure 13. Pinspot and $\mathrm{Cr}$ extension repairs were executed as for a conventional opaque $\mathrm{Cr}$ mask. Pinhole and clear extension repairs were performed using a lower laser power than normal to deposit a thin layer of repair material. Repair quality was assessed using the Aerial Image Measurement System (AIMS ${ }^{\mathrm{TM}}$ ) developed at IBM. ${ }^{8}$ The AIMS ${ }^{\mathrm{TM}}$ instrument was configured to simulate NA $=0.52 \pm 0.02$ and $\sigma=0.59 \pm 0.03$, over a $\pm 1.2 \mu \mathrm{m}$ focal range. Figure 14 shows line scans over a good contact at the various focal positions. Figure 15 shows AIMS ${ }^{\mathrm{TM}}$ images of a contact with $\mathrm{Cr}$ extension before and after repair, and figure 16 shows images of a contact with clear extension before and after repair. The repairs were clear improvements over the defects (with increased depth of focus by as much as $25 \%$ over unrepaired areas), but often they did not duplicate the good 
contact's aerial image. Pinspot repairs appeared most similar to good contacts. Pinhole repairs greater than $\mathrm{k}_{1}=0.5$ away from edges were fine. Clear extension repairs had a nominal impact on $\mathrm{CD}$, but were effective. Cr extension repairs affected both CD and depth of focus and still need work.

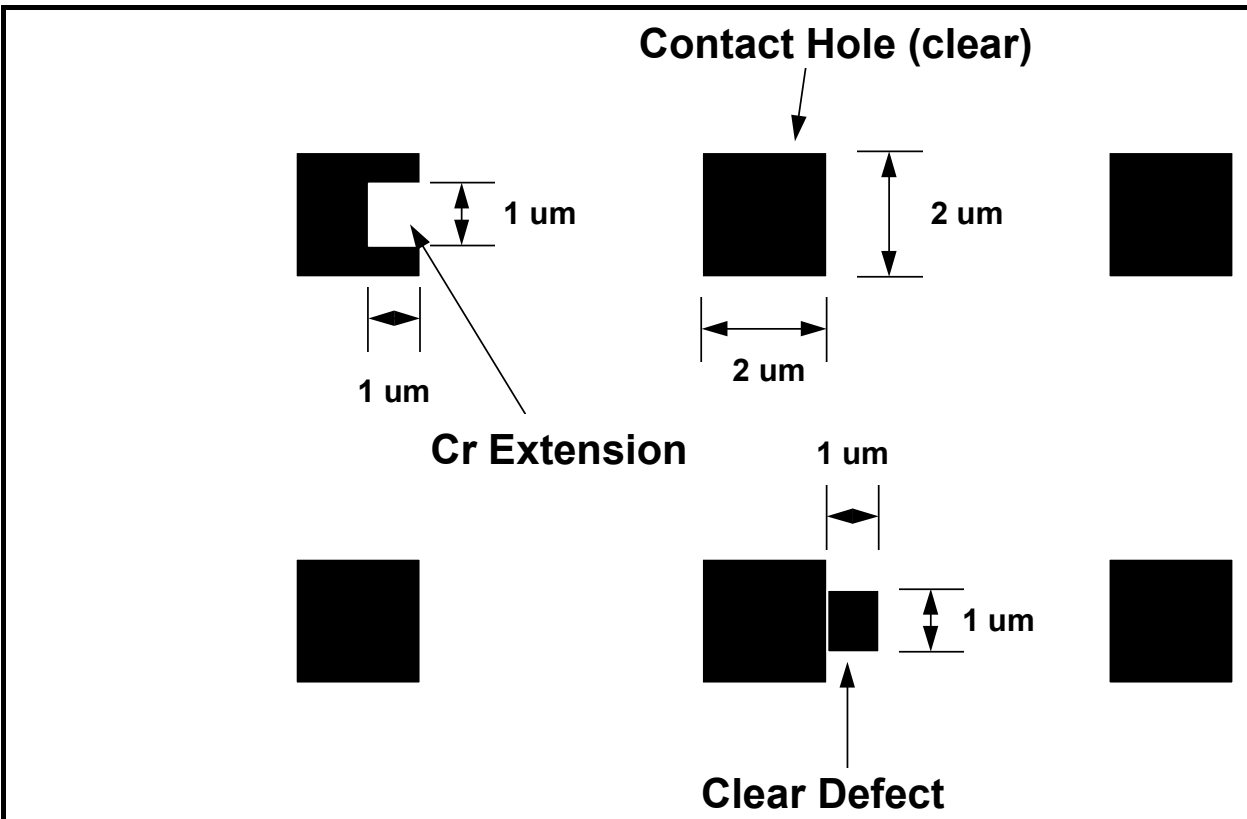

Fig. 13. Schematic diagram of a portion of a contact array with programmed defects (mask dimensions)

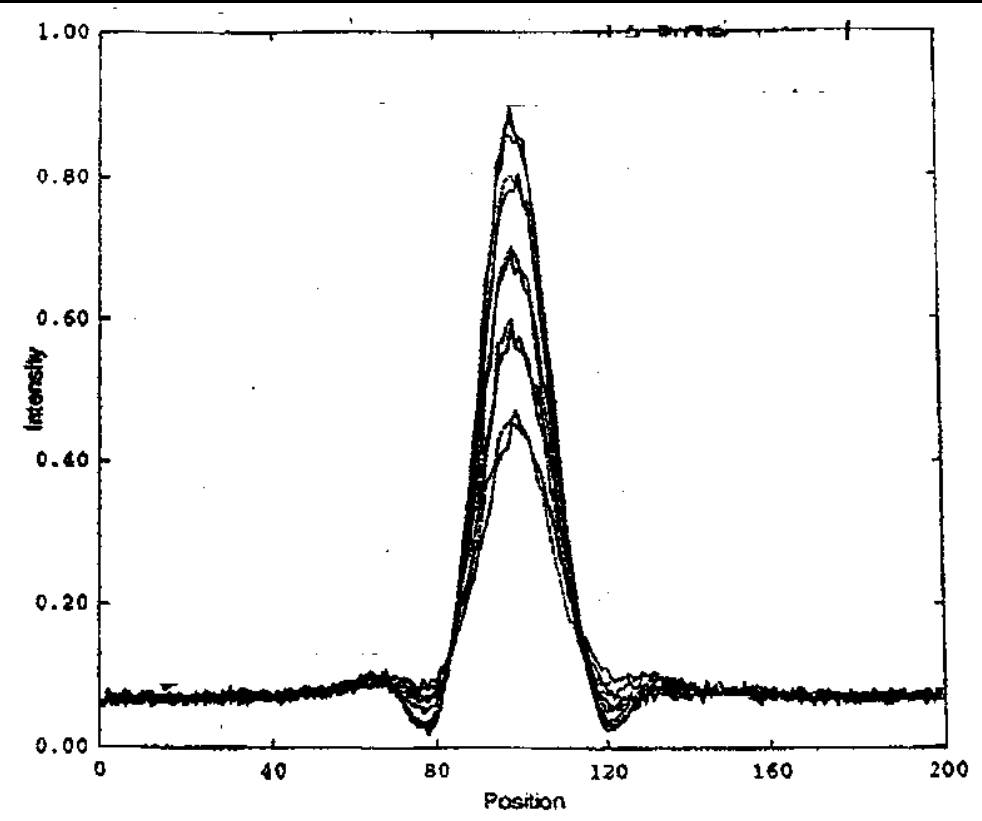

Figure 14. AIMS image of a good $2 \mu \mathrm{m}$ contact (mask dimensions). 


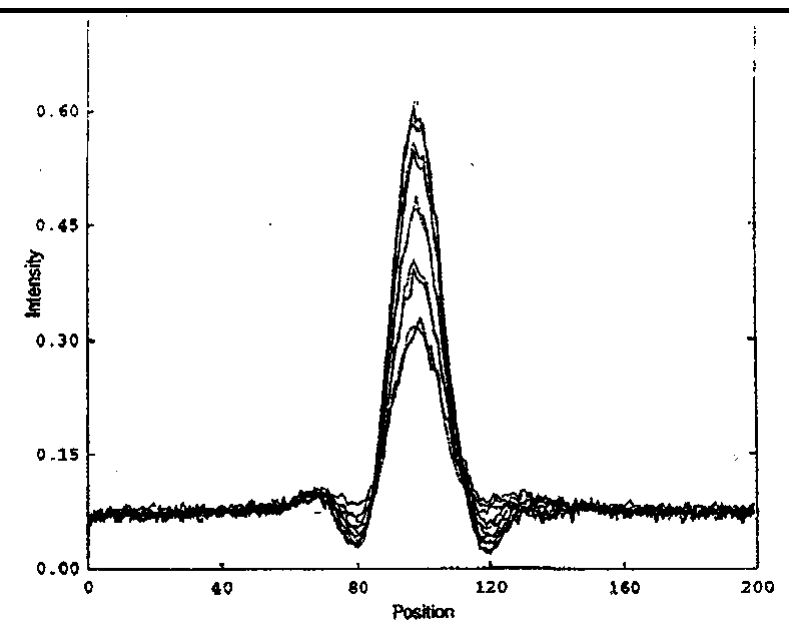

(a)

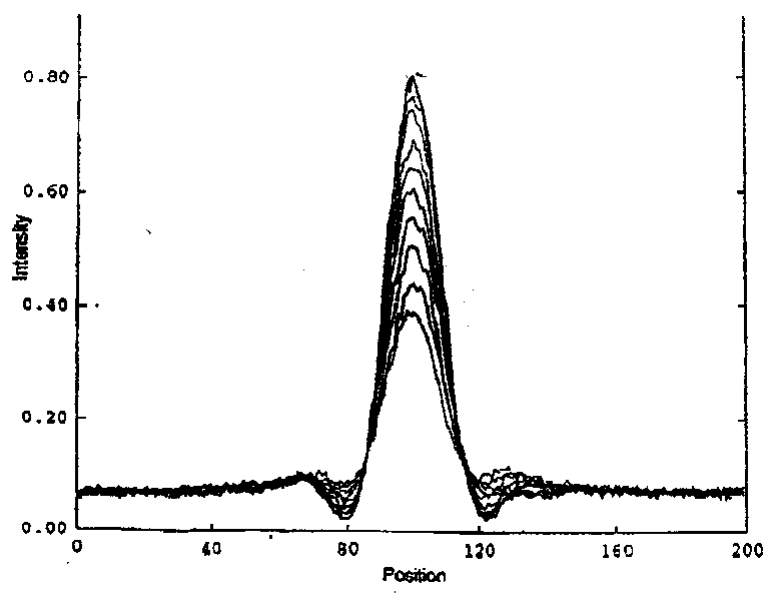

(b)

Fig. 15. AIMS Image of 2 Micron Contact with Chrome Extension:

a) Before Repair (0.61 Peak Intensity)

b) After Repair (0.81 Peak Intensity)

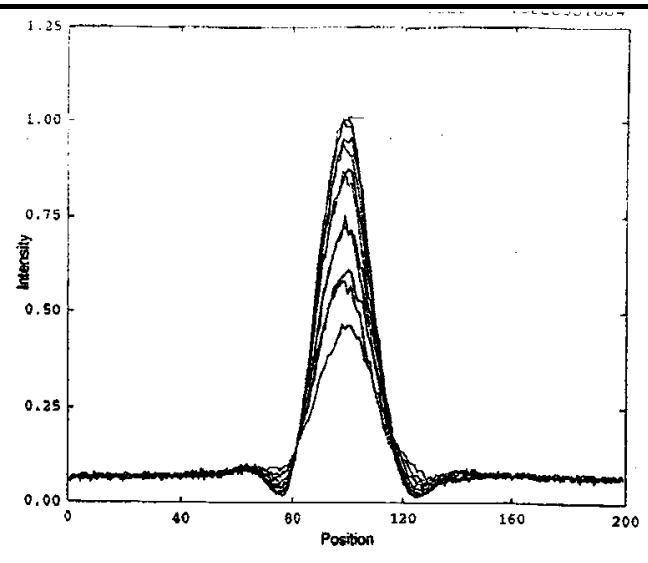

(a)

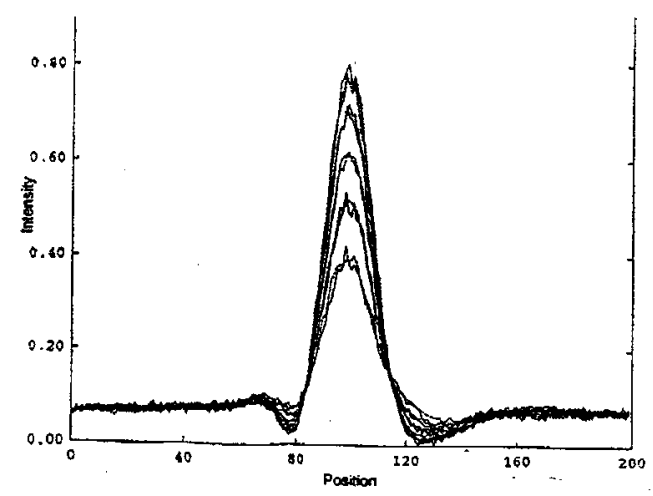

(b)

Fig. 16. AIMS Image of 2 Micron Contact with Clear Extension:

a) Before Repair (1.00 Peak Intensity)

b) After Repair ( Asymmetric Side Lobe, 0.80 Peak Intensity)

\section{LITHOGRAPHY STUDIES}

Cr-based embedded shifter masks are being used or evaluated for several applications. Printing studies have verified predicted performance improvements over conventional masks in cases such as contacts, where attenuated phase shifting masks appear to offer the most potential. ${ }^{9}$ Recent practical work (e.g., printing over topography) has shown that ideal transmissions predicted by modelling can result in excess sidelobe printing, so the useful transmission must be limited. The maximum useful transmission for contacts is about $10 \%$, compared to $12 \%$ or higher predicted by modelling. 
Embedded shifters have been tested on several production aligners. Opaquing frames to eliminate multiple printing at die corners ${ }^{9}$ have been demonstrated. Aligners that separately align the mask and wafer typically use the lithography wavelength to align the mask, and Cr-based materials' compatibility with these systems has been confirmed; little or no alignment system adjustment is required. Systems that align the mask to the wafer use a wavelength that will not expose the photoresist on the wafer, say $633 \mathrm{~nm}$. The embedded shifter's high transmission at $633 \mathrm{~nm}$ may require alignment mark modifications to align properly. One viable solution is to fabricate certain of the alignment structures as subresolution phase gratings, rendering them opaque at the wavelength of interest. Studies of this approach have confirmed its efficacy.

\section{CONCLUSIONS}

Cr-based attenuated photoblanks and masks with $180^{\circ}$ phase shift and flexible optical and physical properties have been developed. They are useful for I-line and G-line lithography and are potentially extendable to DUV. Masks can be processed in a manner very similar to conventional Cr masks, including writing, etching, inspection, and repair. These masks allow improved printing properties for IC manufacturing.

\section{ACKNOWLEDGEMENTS}

The authors thank Sue Garrison for blanks production support, Catherine Wilson for materials modelling, Dennis Swartzfager for SNMS data, Gary Doyle for dry etch work, Tracy Weed of IBM Microelectronics for $257 \mathrm{~nm}$ phase shift measurements, and Ron Martino of IBM Microelectronics for AIMS ${ }^{\mathrm{TM}}$ measurements. This work was partially supported by SEMATECH Contract Number 33005401.

\section{REFERENCES}

1. Burn J. Lin, "The attenuated phase shifting mask," Solid State Technology, Jan. 1992, pp. 43-47.

2. Y.-C. Ku, et. al., "Use of a pi-phase shifting X-ray mask to increase the intensity slope at feature edges," J. Vac. Sci. Technol., Vol. B 6 (1), pp. 150-153, Jan/Feb 1988.

3. Models were developed using WVASE analysis software, provided by J. A. Woollam Company, Lincoln, NE.

4. D. E. Aspnes, "The accurate determination of optical properties by ellipsometry, in Handbook of Optical Constants of

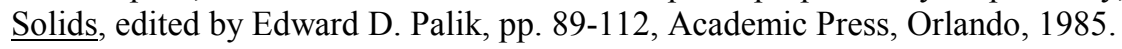

5. CR4 chromium etchant, Cyantek Corp., Fremont, CA.

6. KLA331, KLA Instruments, San Jose, CA.

7. API1100, QC Optics, Inc., Burlington, MA.

8. R. A. Budd, et. al., "A New Phase Shift Mask Aerial Image Measurement and Evaluation Tool, the Microlithography Simulation Microscope," Photomask Japan '94, Kanagawa, April, 1994 (this meeting).

9. M. Yoshioka, et. al., "Practical Attenuated phase-shifting mask with a single layer absorptive shifter of MoSiO and MoSiON for ULSI fabrication," International Electron Devices Meeting, Washington, Dec. 1993.

F. D. Kalk, R. H. French, H. U. Alpay, G. Hughes, SPIE Proceedings Vol. 2254, Photomask Japan 94. 